\title{
Geociências
}

\section{Análise de impactos da metodologia de estimativa da porosidade em reservas de petróleo por meio de simulação de Monte Carlo}

\author{
Gabriel A. C. Lima \\ Laboratório de Análises Geoeconômicas de Recursos Minerais (LAGE) \\ Instituto de Geociências, UNICAMP.Email: gabriel@ige.unicamp.br \\ Alexandre C. Vidal \\ Departamento de Geologia e Recursos Naturais, Instituto de Geociências, UNICAMP. \\ E-mail:vidal@ige.unicamp.br \\ Saul B. Suslick \\ Departamento de Geologia e Recursos Naturais, Instituto de Geociências, UNICAMP. \\ E-mail:suslick@ige.unicamp.br
}

\section{Resumo}

Nos trabalhos de estimativa de reservas de petróleo, geralmente, assume-se que a variável aleatória porosidade do reservatório se distribui de acordo com o modelo Gaussiano. Desse modo, assume-se que o valor esperado da distribuição pode ser estimado por meio da média aritmética dos valores de porosidade obtidos a partir de análises de perfilagens e plugs em nível de poço. Isso implica que a distribuição Gaussiana é a melhor escolha para a modelagem dos dados dessa variável, mas nem sempre essa premissa é verdadeira. Nesse trabalho, ao se utilizarem dados reais de porosidade de uma camada de rocha reservatório do poço 3-NA-002-RJS da bacia de Campos, mostra-se que a distribuição logística é a que melhor se ajusta aos dados amostrais. Em tais casos, como ilustra a análise numérica, pode-se cometer erros significativos ao selecionar a média aritmética amostral como sendo o estimador da média populacional. Os resultados apontam que o uso inadequado do tipo de distribuição pode gerar impactos significativos nas estimativas das reservas recuperáveis, observando-se que a média aritmética tende a subestimar o P90 e a superavaliar o P10.

Palavras-chaves: porosidade, estimativa das incertezas, valor esperado, distribuição de probabilidade.

\begin{abstract}
In many papers dealing with estimation of oil reserves, engineers usually assume that well porosity can be modeled as a Gaussian distribution, that is, under this assumption the expected value of porosity can be estimated from the average porosity values from well $\log$ and petrophysical data. But, other distributions can be used to model local porosity when Gaussian distribution cannot fit sample data. In this paper, using actual porosity data of a 3-NA-002-RJS well from the Campos Basin, it is shown that for a selected interval, the logistic distribution fits the data better than other distributions and its expected value should be used to estimate the well porosities of the entire population. In such cases, as numerical analysis shows, using arithmetic mean instead of expected value may give rise to errors. The data shows that using an average as porosity estimator will overestimate the P90 and underestimate the P10 estimates.
\end{abstract}

Keywords: porosity, uncertainty estimation, expected value, probability distribution. 
Análise de impactos da metodologia de estimativa da porosidade em reservas de petróleo por meio de...

\section{Introdução}

A informação mais importante a ser obtida pelos estudos preliminares de caracterização dos reservatórios é a determinação do volume de óleo in-situ (VOIS). A partir do seu conhecimento, realizam-se estudos no âmbito da engenharia de reservatório com o objetivo de estimar o fator de recuperação e, a seguir, a reserva. De acordo com Chierice (1994, p. 26), o valor esperado do volume da reserva pode ser estimado pelos valores esperados das variáveis presentes na equação abaixo:

$$
\mathrm{E}[\mathrm{R}]=\frac{\mathrm{A}_{\mathrm{m}} * \mathrm{~h}_{\mathrm{m}} * \phi_{\mathrm{m}} *\left(1-\mathrm{S}_{\mathrm{W}_{\mathrm{m}}}\right)}{\mathrm{B}_{\mathrm{o}}} * \mathrm{FR}_{\mathrm{m}}
$$

em que $\mathrm{A}_{\mathrm{m}}$ é a área de drenagem de óleo, $\mathrm{h}_{\mathrm{m}}$ é a espessura das camadas de óleo, $\mathrm{f}_{\mathrm{m}}$ é a porosidade das camadas de óleo, $\mathrm{B}_{\mathrm{o}}$ é o fator volume de formação do óleo, $\mathrm{FR}_{\mathrm{m}}$ é o fator de recuperação e $\mathrm{S}_{\mathrm{Wm}}$ é a saturação de água. Para utilizar a equação (1), faz-se necessário a estimativa de valores médios de seis parâmetros de entrada, os quais, segundo Chierici (1994), são estimados a partir de estudos de sísmica, correlações geológicas e trabalhos de engenharia de reservatório.

Em trabalhos de avaliação de reservas de petróleo, os dados são geralmente escassos, em parte, devido ao seu elevado custo de aquisição, processamento e interpretação. Por isso, entre os poços, naqueles pontos onde o reservatório não é amostrado, faz-se uma estimativa utilizando-se métodos geoestatísticos e métodos clássicos de interpolação. Mas, antes de se realizar os trabalhos de interpolação, deve-se proceder à estimativa dessas variáveis em nível local, ou seja, em cada poço do reservatório. Dessa maneira, as estimativas envolvem grandes níveis de incerteza que começam em nível de poços e, depois, se estendem nas estimativas entre os poços.

Entre as seis variáveis incertas da equação (1), o foco desse artigo consiste em investigar a incerteza presente nos dados de porosidade em nível local de uma camada de rocha-reservatório. A média aritmética verdadeira da porosidade global do reservatório $\left(\phi_{\mathrm{mR}}\right)$ é:
$\phi_{\mathrm{mR}}=\frac{\int_{\mathrm{V}} \phi(\mathrm{x}, \mathrm{y}, \mathrm{z}) \mathrm{dxdydz}}{\int_{\mathrm{V}} \mathrm{dxdydz}}$,

onde $\phi(\mathrm{x}, \mathrm{y}, \mathrm{z})$ é a porosidade em cada ponto do espaço ${ }^{1}$. Ressalta-se que, se fosse possível aplicar a equação (2), o analista estaria calculando uma estatística da população de porosidades do reservatório, ou seja, teria o valor de um parâmetro populacional.

No entanto, na prática, a aplicação da equação (2) geralmente não é possível, pois não há viabilidade econômica e técnica para medir a porosidade de todos os pontos do espaço que delimitam o reservatório. Alternativamente, a média aritmética verdadeira da porosidade global $\left(\phi_{\mathrm{mR}}\right)$ pode ser estimada por meio de:

$\phi_{\mathrm{mR}}=\frac{1}{\mathrm{~N}} \sum_{\mathrm{i}=1}^{\mathrm{N}} \phi_{\mathrm{m}, \mathrm{i}}$

onde $\phi_{\mathrm{m}, \mathrm{i}}$ é a porosidade média em cada $\operatorname{poço}(i=1,2,3, \ldots, N)$. Note que a equação (3) é um estimador da porosidade global a partir de uma amostra coletada em cada poço. Entretanto, antes de usar a equação (3), deve-se, primeiramente, conhecer a porosidade em nível local, ou seja, em cada intervalo de rocha-reservatório (camadas).

Para a estimativa da porosidade, em cada poço, assume-se que ela se comporta como uma variável aleatória, em cada camada desse poço. Na prática, o seu valor esperado pode ser computado por meio de uma média ponderada, considerando-se os seus valores em cada uma das $\mathrm{K}$ camadas que se encontram presentes por meio de:

$\phi_{m, i}=\frac{\sum_{i=1}^{K} \phi_{i} h_{i}}{\sum_{i=1}^{K} h_{i}}$

onde $\phi_{\mathrm{i}}$ é a média aritmética da porosidade em cada uma das k camadas cuja es- pessura individual é $h_{\mathrm{i}}, \mathrm{i}=1$, 2, 3, ..., K. Se a espessura das camadas $\left(h_{i}\right)$ for uniforme, a equação (4) se transforma numa média aritmética.

Ao usar, conjuntamente, as equações (3) e (4) pode-se obter estimativas da porosidade global do reservatório e de cada poço. No entanto, cabe uma ressalva. Essas equações fornecem resultados não tendenciosos desde que a variável aleatória porosidade tenha uma distribuição simétrica, tal como a Gaussiana. Essa parece ser uma premissa freqüentemente usada nos trabalhos de avaliação de campos de petróleo, pois ela é compatível com Deutsch (2002), ao afirmar que, na prática da indústria de petróleo, geralmente a média aritmética amostral é usada como a estatística que representa o valor esperado da distribuição de valores de porosidade.

Por outro lado, pode ocorrer que a distribuição Gaussiana não seja a que melhor se ajusta aos dados de porosidade de uma determinada camada de rocha-reservatório ou poço. A investigação sobre a distribuição probabilística para a modelagem dos dados de porosidade é a principal motivação desse artigo. Além disso, faz-se uma análise de possíveis erros de estimativa da reserva recuperável por meio das medidas de risco (P10, P50 e P90) para situações em que uma distribuição assimétrica seja mais apropriada para a modelagem dos mencionados dados de porosidade.

Esse artigo é organizado em três partes. A primeira parte apresenta a metodologia para a estimativa da porosidade em nível de poço. A segunda parte descreve uma aplicação dessa metodologia aos dados de porosidade de uma camada de rocha-reservatório do poço 3-NA-0002-RJS. A parte final apresenta as principais conclusões do artigo.

\footnotetext{
${ }^{1}$ Na verdade, a equação (2) pode ser usada para calcular a média aritmética verdadeira em qualquer espaço e não apenas obter uma estimativa.
} 


\section{Metodologia para a estimativa da porosidade do poço}

A estimativa da porosidade verdadeira, em nível de camada de rocha-reservatório que é atravessada pelos poços, deve ser realizada por intermédio da análise estatística dos dados amostrais. A escolha do valor que representa a porosidade, em cada camada de rocha-reservatório no poço, geralmente situa-se entre a média, a mediana e a moda da distribuição. No caso de distribuições simétricas (por exemplo, a distribuição normal), essas três medidas de tendência central são iguais, mas, no caso de distribuição assimétrica (por exemplo, a lognormal, Weibull, etc), essas medidas são diferentes.

Assim, inicialmente, deve-se realizar a escolha da distribuição que seja compatível com os valores que compõem a amostra de porosidade, ou seja, não se devem ajustar os dados à distribuição de probabilidade. Para tal procedimento, não há uma regra rígida, mas consideram-se, entre outros, os seguintes critérios:

1. A natureza física da variável e, conseqüentemente, os valores numéricos que ela pode assumir. Por exemplo, o modelo matemático da porosidade só pode assumir valores entre zero e $100 \%$, não podendo contemplar valores negativos ou superiores à unidade. Logo, teoricamente, a distribuição Gaussiana sem truncamento é inadequada ${ }^{2}$ para a modelagem de porosidade, pois as variáveis aleatórias dessa distribuição assumem valores negativos e positivos.

2. Um outro critério refere-se à dinâmica do tipo de interação (aditiva, multiplicativa, ou mista) entre os agentes físicos e químicos que atuam conjuntamente ou isoladamente para a formação da porosidade. Se esses agentes agirem aditivamente, podese pensar que uma distribuição simétrica seja apropriada; já se eles agirem multiplicativamente, pode-se pensar em alguma distribuição assimétrica.
3. Em muitos casos, algumas distribuições já são bastante estudadas na literatura, de modo que a experiência pode ser um elemento adicional para contribuir para a escolha, como mostram Fowler et al. (1999). No entanto, deve-se ter cautela ao aceitar sugestões de modelos-padrões, pois o caso particular pode ser diferente de um modelo global.

4. No processo de escolha da distribuição, deve-se conhecer os valores da variável que possuem significado físico. Por exemplo, ao ajustar uma distribuição não estável ${ }^{3}$ para modelar os dados de porosidade, não se pode esperar usar o valor esperado para caracterizar a porosidade local porque essa distribuição possui valor esperado infinito. Lévy (1925), Andrews (1991), Barndorff-Nielsen et al. (2001) e Applebaum (2004) apresentam alguns detalhes sobre as distribuições estáveis Gaussianas e nãoGaussianas.

Após considerar os fatores acima e escolher a distribuição de probabilidade, deve-se estimar os seus parâmetros a partir das estatísticas dos dados de porosidade disponíveis. De acordo com Bhattacharyya e Johnson (1977), há diversos métodos para a estimativa dos parâmetros, entre eles a máxima verossimilhança (MLE), regressão linear, etc. Embora os estimadores de MLE possam ser tendenciosos, se o tamanho da amostra for pequeno, nesse trabalho considerar-se-á a MLE 4 , uma vez que esse método usa somente características matemáticas da distribuição (equação da função densidade de probabilidade), como mostram Hoog e Craig (1995), Ai (1997) e Härdle et al. (2004).

Em teoria, podem-se ajustar diferentes distribuições ao mesmo conjunto de dados, mas elas terão graus de ajustamento distintos. Assim, após a escolha das distribuições candidatas em acordo com os quatro itens discutidos anteriormente, deve-se realizar uma análise de aderência aos dados amostrais. Os principais testes são Anderson-Darling (A-D), Qui-quadrado e KolmorogovSmirnov (K-S). O teste de qui-quadra- do ${ }^{6}$ é usado para testar o ajuste de uma distribuição aos dados amostrais, mas se aplica ao caso de dados discretos e seus resultados são dependentes da escolha do número de classes. O teste de Kolmorogov Smirnov (K-S) ${ }^{7}$, por sua vez, é independente do número de classes, mas ele se concentra em comparar o centro dos dados amostrais como o centro de uma distribuição teórica, não cap-

${ }^{2}$ Naturalmente, esse problema pode ser contornado pela escolha de uma distribuição normal truncada.

${ }^{3}$ Distribuições estáveis são aquelas que possuem momentos finitos sob adição, ou seja, a soma de $\mathrm{N}$ variáveis estáveis é uma variável aleatória estável, tal como a distribuição Gaussiana. Por exemplo, a distribuição de Cauchy possui valor esperado infinito, de modo que ela é teoricamente inadequada para a modelagem da variável porosidade.

${ }^{4}$ Ressalta-se que, para a distribuição normal, os estimadores não-tendenciosos dos dois parâmetros da distribuição são:

$\mu=\frac{1}{N} \sum_{i=1}^{N} X_{i}$ e $\sigma^{2}=\frac{1}{(N-1)} \sum_{i=1}^{N}\left(X_{i}-\bar{X}\right)^{2}$. Ver Bhattacharyya e Johnson (1977).

${ }^{5} \mathrm{O}$ método da MLE é independente de qualquer posição dos dados amostrais de porosidade, tal como ocorre com a regressão linear.

${ }^{6}$ A equação do qui-quadrado é: $\mathrm{X}^{2}=\sum_{\mathrm{i}=1}^{\mathrm{k}} \frac{\left(\mathrm{N}_{\mathrm{i}}-\mathrm{E}_{\mathrm{i}}\right)}{\mathrm{E}_{\mathrm{i}}}$, onde ké o número de classes, $\mathrm{N}_{\mathrm{i}}$ é o número de dados dentro de uma classe, $E_{\mathrm{i}}$ é a quantidade de dados que deve estar presente na classe segundo a distribuição teórica.

${ }^{7} \mathrm{O}$ teste de K-S é dado por meio de:

$\mathrm{K}-\mathrm{S}=\sup \left[\left|\mathrm{F}_{\mathrm{n}}(\mathrm{x})-\hat{\mathrm{F}(\mathrm{x})}\right|\right], \mathrm{F}_{\mathrm{n}}(\mathrm{x})$ é a fração de amostras acima de uma valor n e $\hat{\mathrm{F}}(\mathrm{x})$ é o número de amostras esperado para a distribuição teórica. 
Análise de impactos da metodologia de estimativa da porosidade em reservas de petróleo por meio de...

turando, assim, as discrepâncias nas extremidades, ou seja, praticamente ignora os dados extremos, o que pode ser crítico ao estudar distribuições fortemente assimétricas. O teste de AndersonDarling8 (A-D) também é independente do número de classes e se concentra em medir se há um bom ajuste nos extremos da distribuição, sendo recomendado para o caso de distribuiçõos assimétricas.

Ao analisar as características desses testes, nota-se que a informação de cada um deles é limitada, pois ora medem o ajuste do centro da curva aos dados amostrais, ora o das extremidades, não considerando, ainda, o problema "quase” subjetivo da escolha do número de classes, no caso do teste do quiquadrado - para mais detalhes, consultar Law e Kelton (1982). Apesar de suas limitações em relação ao tamanho da amostra, nesse artigo utilizar-se-á o teste de K-S para escolher a distribuição, uma vez que este busca identificar aquela que melhor se ajusta aos valores centrais das amostras. Isto porque, como os dados de porosidade são oriundos de um intervalo de rocha sedimentar que possui uma litologia relativamente "homogênea" no intervalo amostrado, espera-se que os dados não sejam discrepantes.

\section{Resultados e discussões sobre porosidade do Poço 3 NA-0002-RJS}

Os dados foram retirados do poço NA-002-RJS situado no Campo de Namorado, bacia de Campos. Esse poço atravessa um reservatório produtor que consiste em depósitos de areias turbidíticas depositadas durante o AlbianoCenomaniano, como descrito em Guardado et al. (1989). A camada se encontra à profundidade de 3.041,7 metros e com espessura de 12,5 metros. Os dados de porosidade são referentes à camada mais espessa de rocha-reservatório e se constituem em 35 valores obtidos por testemunho (petrofísica) e 82 valores obtidos por perfis.
Sobre a técnica de aquisição de dados de porosidade, na prática, eles são determinados por meio de dois métodos distintos: 1) pela interpretação dos dados de perfis geofísicos de poços (medida indireta de porosidade) e, 2) por meio de medidas de petrofísica (medida direta de porosidade).

O primeiro conjunto de dados foi obtido por meio da análise de perfil gerado por medidas geofísicas (densidade, sônico e neutrão). Entre estes, segundo Schlumberger (1987), o perfil de densidade é o mais apropriado para a medição da densidade da rocha, pois as medições são realizadas através da densidade da rocha-matriz e do fluido. A relação entre densidade da rocha-matriz, densidade de fluido e porosidade é:

$$
\phi=\frac{\rho m a-\rho b}{\rho m a-\rho f l}
$$

onde a densidade relativa da matriz é $\rho_{m a}$, a densidade lida no aparelho é $\rho_{b}$ e a densidade relativa do fluido é $\rho_{f l}$. Nesse artigo, considera-se que, para o arenito, tem-se que $\rho_{\text {ma }}=2,65$ e que $\rho_{f l}=1,05$.

Um dos fatores determinantes da incerteza nas medidas de porosidade a partir de perfis é o valor verdadeiro de densidade da matriz. De acordo com Schlumberger (1987), esses valores variam usualmente entre 2,65 a 2,85g/ $\mathrm{cm}^{3}$, dependendo da composição da litologia da rocha. Nesse contexto, Lerche (1997) e Abrahamsen et al. (2000) afirmam que, como dificilmente uma determinada rocha possui composição litológica constante, ao longo de uma camada, há uma grande incerteza sobre o valor real da densidade da rocha-matriz. Outra fonte de incerteza está relacionada à densidade do fluido, que apresenta relação direta com a concentração de cloreto na água de formação. Entretanto, em escala de reservatório, a incerteza relacionada a esta variável pode ser considerada desprezível, como argumentam Lake e Carroll (1986). Finalmente há a incerteza da medição instrumental, que geralmente é considerada de 5\%, como atestam Hearst e Nelson (2000), Mann (1993) e Bárdossy e Fodor (2001).
Um segundo conjunto de dados se refere às informações obtidas por meio de análise de petrofísica, baseada nas medidas de porosímetros, sendo que cada resultado representa o valor de porosidade de um plugue com dimensões de alguns centímetros (em torno de $4 \mathrm{~cm}$ ). Os dados de porosidade analisados nesse trabalho se encontram nas Tabelas 1 e 2. Primeiramente, na Tabela 1 , encontram-se os 81 valores obtidos por meio da análise de perfis de densidade da camada de rocha-reservatório atravessada pelo poço 3-NA-0002-RJS.

Os valores das amostras que se encontram na Tabela 1 possuem uma variabilidade razoável, pois o menor valor é 23,03\% e o maior é 34,94\%. Utilizando-se a equação (4) e considerando-se uma distribuição espacial uniforme dessas amostras, a média aritmética da porosidade da camada de rocha-reservatório atravessada pelo poço é 31,83\% e o desvio-padrão é de 1,75 \%.

Além dos dados de porosidade obtidos por perfis de densidade, foram coletadas amostras para serem submetidas a testes de petrofísica. A amostra composta pelos 33 valores de porosidade obtidos por petrofísica se encontra na Tabela 2.

O tamanho da amostra de dados de porosidade obtidos por petrofísica é menor que o da amostra de dados obtidos a partir de perfis de densidade, mas eles se referem à porosidade efetiva da rocha. No entanto, nesses dados, os geólogos possuem um nível mais elevado

${ }^{8}$ A equação do teste de Anderson-Darling é:A-D $=n \int_{-\infty}^{+\infty}[F(x)-\hat{F}(x)]^{2} \psi(X) \hat{f}(X) d X$,
onde $\psi^{2}=\frac{1}{\hat{F}(X)[1-F(X)]}, f(X)$ é a função densidade de probabilidade que é candidata a modelar a população, $\mathrm{F}_{\mathrm{n}}(\mathrm{X})=\mathrm{N}_{\mathrm{X}} / \mathrm{n}, \mathrm{N}(\mathrm{X})$ é a quantidade de dados abaixo de $\mathrm{X}$. 
Gabriel A. C. Lima et al.

de confiança que naqueles obtidos por perfis para fazer inferência sobre as características das rochas. Nesse caso, a média aritmética da porosidade é $25,86 \%$ e o desvio-padrão é 4,45\%, ou seja, os dados de porosidade de petrofísica possuem maior variabilidade.

Os valores de porosidade obtidos pelos dois métodos apresentam características diferentes, pois, na verdade, medem grandezas distintas. Os perfis densidade medem a porosidade total, enquanto que as análises de petrofísica medem a porosidade efetiva. Ademais, os dados provenientes de perfil foram coletados segundo um espaçamento de 20 cm e, por isso, há uma elevada quantidade de informação. Já o espaçamento de coleta dos plugues, a partir dos quais se estima a porosidade, é irregular. Ressalta-se que, na prática, não há regras claras para a definição do número de amostras destinadas a petrofísica ou o espaçamento entre as amostras, de modo que a decisão sobre o procedimento a ser adotado para os ensaios petrofísicos depende do objetivo do trabalho a ser desenvolvido conforme ressaltam Salomão e Grell (2001).

A partir desses dados, a estimativa da porosidade local baseada na teoria tradicional consiste em admitir que a distribuição Gaussiana é a que melhor se ajusta aos dados das Tabelas 1 e 2. Mas, ao aceitar passivamente essa premissa, pode-se gerar erros nos casos em que uma distribuição não-Gaussiana for mais apropriada. Por isso, nesse trabalho, fazse uma análise mais aprofundada, visando a obter a melhor distribuição para a modelagem dos dados existentes. A distribuição de probabilidade logística é a escolhida pelo critério de aderência de K-S (Kolmorogov-Smirnov) e os seus parâmetros ( $\alpha$ e $\beta$ ) foram estimados por meio da MLE. Os resultados encontramse na Tabela 3.

A distribuição logística é a mais apropriada para modelar as populações que deram origem aos valores de porosidade, tanto para o caso de dados obtidos por perfilagem como por petrofísica. Para o conjunto de dados de porosidade
Tabela 1 - Valores de porosidade obtidos por informação de perfil na camada de rocha-reservatório do poço 3-NA-0002-RJS.

\begin{tabular}{l|l|l|l|l|l}
\hline $34,44 \%$ & $33,67 \%$ & $29,94 \%$ & $32,57 \%$ & $32,28 \%$ & $3,44 \%$ \\
\hline $34,94 \%$ & $33,70 \%$ & $28,84 \%$ & $32,02 \%$ & $32,26 \%$ & $30,75 \%$ \\
\hline $34,87 \%$ & $34,10 \%$ & $27,74 \%$ & $31,20 \%$ & $32,29 \%$ & $31,33 \%$ \\
\hline $34,05 \%$ & $33,95 \%$ & $26,31 \%$ & $31,06 \%$ & $32,60 \%$ & $30,85 \%$ \\
\hline $33,84 \%$ & $33,42 \%$ & $29,89 \%$ & $31,03 \%$ & $32,44 \%$ & $30,65 \%$ \\
\hline $34,19 \%$ & $33,25 \%$ & $31,70 \%$ & $30,99 \%$ & $32,27 \%$ & $30,45 \%$ \\
\hline $34,31 \%$ & $33,58 \%$ & $32,28 \%$ & $31,12 \%$ & $31,79 \%$ & $30,91 \%$ \\
\hline $34,04 \%$ & $32,77 \%$ & $32,86 \%$ & $31,36 \%$ & $31,30 \%$ & $31,52 \%$ \\
\hline $33,91 \%$ & $31,92 \%$ & $33,31 \%$ & $31,61 \%$ & $30,33 \%$ & $31,50 \%$ \\
\hline $33,79 \%$ & $31,06 \%$ & $33,12 \%$ & $32,30 \%$ & $30,04 \%$ & $31,35 \%$ \\
\hline $31,19 \%$ & $31,36 \%$ & $31,04 \%$ & $31,56 \%$ & $30,14 \%$ & $31,92 \%$ \\
\hline $31,15 \%$ & $31,71 \%$ & $30,93 \%$ & $31,56 \%$ & $29,79 \%$ & $32,29 \%$ \\
\hline $29,43 \%$ & $32,69 \%$ & $30,58 \%$ & $23,58 \%$ & $30,97 \%$ & $23,03 \%$ \\
\hline $29,74 \%$ & $32,89 \%$ & $22,49 \%$ & & & \\
\hline
\end{tabular}

Tabela 2 - Dados de porosidade obtidos por petrofísica na camada de rochareservatório do poço 3-NA-0002-RJS.

\begin{tabular}{l|l|l|l|l}
\hline $27,10 \%$ & $22,20 \%$ & $27,20 \%$ & $26,50 \%$ & $28,40 \%$ \\
\hline $23,90 \%$ & $23,30 \%$ & $23,60 \%$ & $28,10 \%$ & $27,40 \%$ \\
\hline $28,20 \%$ & $26,70 \%$ & $25,70 \%$ & $30,20 \%$ & $28,20 \%$ \\
\hline $24,00 \%$ & $23,20 \%$ & $25,80 \%$ & $27,10 \%$ & $25,10 \%$ \\
\hline $22,60 \%$ & $26,30 \%$ & $28,20 \%$ & $29,80 \%$ & $24,70 \%$ \\
\hline $29,80 \%$ & $29,70 \%$ & $29,10 \%$ & & \\
\hline
\end{tabular}

Tabela 3 - Distribuições selecionadas para a modelagem da porosidade.

\begin{tabular}{|c|c|c|}
\hline $\begin{array}{c}\text { Origem dos } \\
\text { dados }\end{array}$ & $\begin{array}{l}\text { Distribuição } \\
\text { selecionada }\end{array}$ & $\begin{array}{l}\text { Parâmetros da distribuição: } \\
\alpha=\text { localização e } \beta=\text { escala }\end{array}$ \\
\hline Perfil & Logística & $\begin{array}{c}\alpha=31,73 \% \\
\beta=1,100 \\
\text { Mediana }=\text { moda }=\text { valor esperado }=31,73 \% \\
\text { Assimetria }=0 \\
\text { Curtose }=4,2\end{array}$ \\
\hline Petrofísica & Logística & $\begin{array}{c}\alpha=26,65 \% \\
\beta=1,780 \\
\text { Mediana }=\text { moda }=\text { valor esperado }=26,64 \% \\
\text { Assimetria }=0 \\
\text { Curtose }=4,2\end{array}$ \\
\hline
\end{tabular}


Análise de impactos da metodologia de estimativa da porosidade em reservas de petróleo por meio de...

de petrofísica e perfil, os valores dos parâmetros da distribuição logística mostram que ela é uma distribuição simétrica (assimetria é zero), mas possui um achatamento diferente da distribuição nor$\mathrm{mal}^{9}$. Assim, o valor esperado ${ }^{10}$ da porosidade na camada de rocha é $31,73 \%$, enquanto que a média aritmética é $31,83 \%$. Pode-se observar que, embora para esses dados a distribuição logística seja simétrica, o seu valor esperado não é igual à média aritmética ${ }^{11}$ da amostra, pois as distribuições logísticas e normal são diferentes - a curtose da Gaussiana é 3 , enquanto que, para esses dados, a curtose da logística é 4,2.

No entanto, à medida que a distribuição se torna mais assimétrica, a diferença aumenta, como mostram Costa Lima, Vidal e Suslick (2005). Para ilustrar tais diferenças entre média aritmética e valor esperado, consideram-se os valores hipotéticos de porosidade de uma camada de rocha-reservatório que se encontram na Tabela 4.

A média aritmética da amostra de dados de porosidade é $29,03 \%$. Ao utilizar esse indicador para representar toda uma população, admite-se, implicitamente, que a distribuição é Gaussiana, isto é, significa que $50 \%$ dos valores se encontram abaixo de $29,03 \%$, enquanto que os outros $50 \%$ se encontram acima, ou seja, a média aritmética coincide com a mediana. No entanto, ao se analisarem os dados da Tabela 4, nota-se que há, não só uma concentração próxima a 26,0\%, mas, também, poucos valores muito altos, acima de 50,0\%. Logo, para esses casos, a média aritmética pode ser diferente da mediana.

Ao se executar o teste de aderência de K-S e ao se usar a MLE, para a estimativa de parâmetros populacionais, a dis- tribuição log-logística é sugerida, ou seja, uma distribuição assimétrica cujo valor esperado é de $27,85 \%$ e mediana de $25,94 \%$. Esses resultados mostram que a média aritmética pode ser um estimador tendencioso da média populacional e, na prática, pode ocasionar grandes erros. Logo, nem sempre é correto utilizar a média aritmética para estimar o valor esperado.

Apesar da pequena diferença $(0,1 \%)$ entre a média aritmética $(31,83 \%)$ e valor esperado da distribuição logística $(31,73 \%)$, nos dados de porosidade de perfil na Tabela 2, essa discrepância pode assumir proporções significativas em decorrência das incertezas nas estimativas de reserva. Uma análise similar pode ser realizada ao serem considerados os dados de amostra de porosidade obtidos por meio dos plugues.

Para ilustrar os erros, ao se escolher a média aritmética como premissa, numa estimativa de reserva, considere uma reserva hipotética que possui somente uma camada de rocha-reservatório e é atravessada pelo poço 3 NA-0002RJS, na qual a porosidade seja a variável incerta. Outras informações desse reservatório são: área do reservatório igual a $1.000 .000 \mathrm{~m}^{2}$, espessura média da camada igual a 12,5 m; saturação de água igual a 20\% e fator de recuperação igual a 15\%.

Como premissa preliminar, considere-se que a porosidade será estimada utilizando-se os dados de petrofísica da Tabela 2. Como discutido em parágrafos anteriores nesse artigo, há duas abordagens distintas ${ }^{12}$ para estimá-la, que são comumente usadas. A primeira é estimar a média populacional por meio da média aritmética, ou seja, 25,86\%. A segunda consiste em estimar o valor esperado da distribuição logística, ou seja, 26,65\%.

Tabela 4 - Dados hipotéticos de porosidade de uma camada de arenito.

\begin{tabular}{c|c|c|c|c}
\hline $25,00 \%$ & $30,18 \%$ & $25,71 \%$ & $26,00 \%$ & $51,00 \%$ \\
\hline $24,00 \%$ & $25,00 \%$ & $25,81 \%$ & $20,00 \%$ & $54,00 \%$ \\
\hline $23,10 \%$ & $26,00 \%$ & $24,00 \%$ & $23,00 \%$ & - \\
\hline $24,11 \%$ & $26,00 \%$ & $26,00 \%$ & $27,00 \%$ & - \\
\hline $24,74 \%$ & $26,00 \%$ & $28,97 \%$ & $53,08 \%$ & - \\
\hline
\end{tabular}

${ }^{9}$ A distribuição normal possui assimetria igual a 0 e curtose igual a 3 .

${ }^{10}$ Ressalta-se aqui que o valor esperado da porosidade é um parâmetro da população, enquanto que a média aritmética é o seu estimador não tendencioso somente no caso em que a variável porosidade tiver uma distribuição Gaussiana.

${ }^{11}$ Para a distribuição logística, o valor esperado da porosidade é estimado por meio de $\mathrm{E}[\phi]=$ a e sua variância é $\operatorname{Var}[\phi]=\frac{1}{2}(\Pi \beta)^{2}$.

${ }^{12}$ Apenas citamos que na prática são usadas essas duas abordagens, mas não afirmamos que as duas são sempre corretas. 
Gabriel A. C. Lima et al.

(P90) obtidos da curva função de probabilidade acumulada decrescente. Essa abordagem facilita as escolhas, pois permite quantificar a exposição ao risco dos investidores.

Para ilustrar o impacto da incerteza da porosidade local numa estimativa de reserva, vamos estimar a curva de probabilidade de reserva por meio de simulação de Monte Carlo para duas premissas:

a) A porosidade se distribui de acordo com uma distribuição Gaussiana com média de 25,86\% e variância de $10,48 \%$.

b) A distribuição da porosidade local é log-logística cuja função densidade de probabilidade é:

$$
f(f)=\frac{1}{\beta} \frac{e^{\left(\frac{\phi-\alpha}{\beta}\right)}}{\left.1+e^{\left(\frac{\phi-\alpha}{\beta}\right)}\right)}
$$

onde os parâmetros $\alpha=26,650$ e $\beta=1,785$ foram estimados pelo método da MLE. O leitor interessado em mais detalhes sobre a distribuição logística pode consultar Law e Kelton (1982).

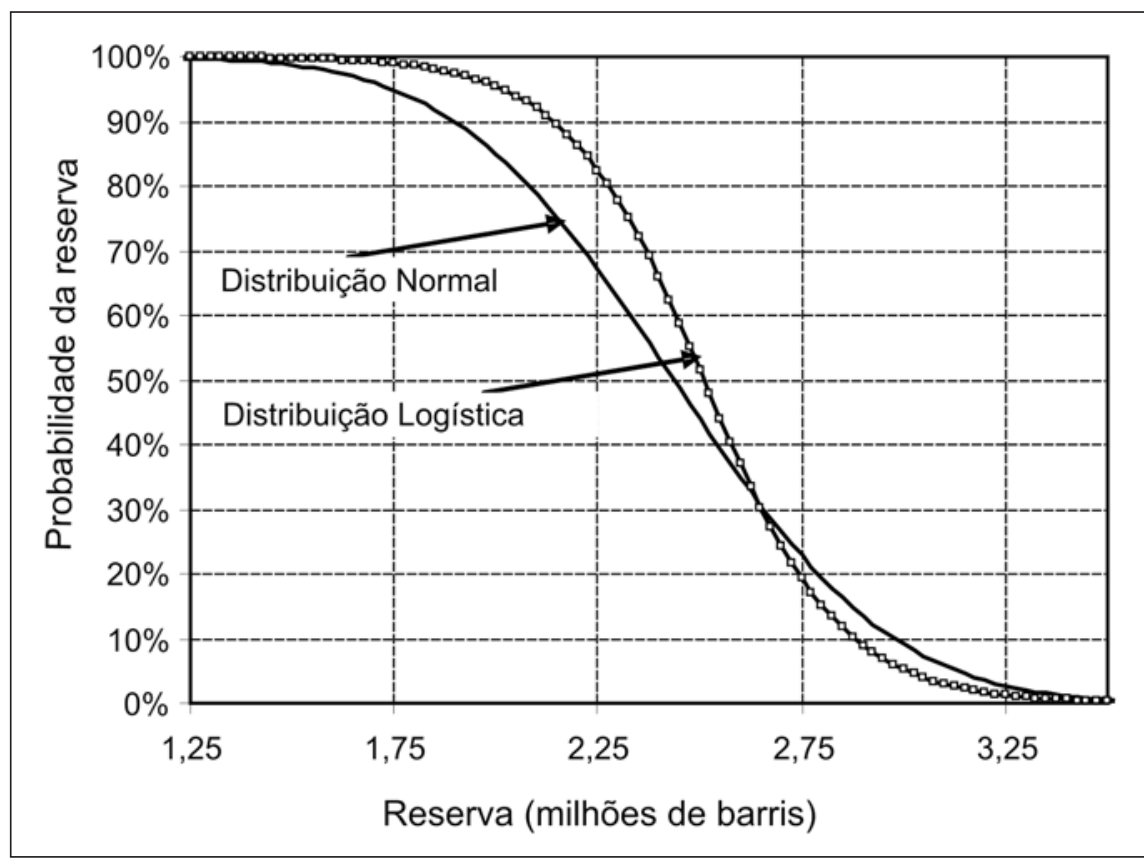

Figura 1 - Curva de risco da reserva de petróleo para duas distribuições para a porosidade.
Ao se realizar uma simulação com 10 mil iterações, obtém-se a função de probabilidade acumulada decrescente ou curva de risco do volume da reserva de petróleo que se encontra na Figura 1.

Na Figura 1, nota-se que a forma da curva da função de probabilidade acumulada decrescente da reserva depende da escolha da distribuição de probabilidade local: se Gaussiana ou logística. Ao se escolher a distribuição Gaussiana, assume que o valor esperado da população de porosidade é estimado por meio da média aritmética. Por outro lado, ao se escolher a distribuição logística, a média aritmética não deve ser usada para a estimativa do valor esperado da população de porosidade.

Analisando-se essas duas curvas, pode-se quantificar os impactos nas estimativas do P90, P10 e P50 do volume de óleo ${ }^{13}$, ao se escolher a premissa de que os valores de porosidade se distribuem segundo uma distribuição Gaussiana e logística. Ao se escolher a última distribuição, nota-se que do P100 até o P30, a média aritmética subavalia o volume de óleo da reserva. Abaixo de P30, a óleo da reserva. média aritmética superavalia o volume de
Por exemplo, o P90 significa que há $90 \%$ de chance de que o valor real da reserva seja de, no mínimo, 1,8 milhões de barris (distribuição Gaussiana) e 2,3 milhões de barris (distribuição logística). Essa disparidade deve-se à escolha do modelo probabilístico para descrever a população dos valores de porosidade em nível local. Nesse caso, o P90 estimado, segundo a suposição da distribuição Gaussiana, seria subavaliado em 21,748\%. Um comportamento similar acontece no caso do P50. Já no caso do P10, ocorre o contrário, havendo 10\% de chance de que o valor mínimo da reserva seja de 2,87 milhões de barris (distribuição logística) e 3 milhões de barris (distribuição normal). Nesse caso, a suposição de distribuição Gaussiana subavalia a reserva em 4,3\%. Ressalta-se que, na prática da indústria de petróleo, esta abordagem para a estimativa do nível de risco das reservas deve ser considerada dentro de uma determinada estratégia de produção. Por exemplo, a mudança das estratégias de produção de um campo (elevar a produção inicial ou retardá-la) provoca uma alteração do nível de risco do projeto.

Finalmente, vale ressaltar que as informações utilizadas nesse trabalho foram provenientes de um estudo detalhado de descrição de testemunhos, em um campo de petróleo com alta densidade de informação. Certamente em situação com menor densidade de informação e menor controle geológico, esta análise pode apresentar resultados diferentes, com maior variabilidade das amostras e maiores desvios em relação aos valores médios. Isso tende a reforçar o argumento de que a análise criteriosa do modelo probabilístico para a modelagem da porosidade em nível local é de fundamental importância para estimar a reserva de petróleo.

${ }^{13}$ De uma forma geral, o PX é o valor de reserva $\mathrm{R}$ tal que haja $\mathrm{X} \%$ de probabilidade de que o valor mínimo da reserva seja igual a $\mathrm{R}^{*}$. 
Análise de impactos da metodologia de estimativa da porosidade em reservas de petróleo por meio de...

\section{Conclusões}

Nesse artigo, foram analisados os dados de porosidade de uma camada de rocha-reservatório atravessada pelo poço 3-NA-0002-RJS do Campo de Namorado, cujas informações são usadas para a estimativa da reserva recuperável de petróleo. Os dados mostram que a porosidade é uma variável aleatória, cujos valores oscilam ao longo do espaço e, por isso, torna-se difícil estimar um único valor representativo para todos aqueles de uma camada.

Para os dados que foram investigados, constatou-se que a distribuição normal não é a mais apropriada para modelar a população de porosidade em nível de poço, mas, sim, a distribuição logística. Portanto a média aritmética é uma medida que pode ser tendenciosa para representar a porosidade em nível local.

Alguns dos impactos da escolha da distribuição de probabilidade em nível local na distribuição de probabildade da reserva foram estudados por meio de um exemplo numérico. Observou-se que uso inadequado da distribuição Gaussiana (ao invés da distribuição logística) pode superavaliar o P90 e subestimar o P10. Isso confirma, mais uma vez, que se deve fazer uma análise estatística dos dados, de modo que possa esolher a melhor distribuição para modelar a população que dá origem à amostra de dados de porosidade em nível local.

\section{Agradecimentos}

Os autores gostariam de agradecer o apoio do CNPq, FAPESP e do Centro de Estudos de Petróleo-CEPETRO da Unicamp para a realização dessa pesquisa. Agradecemos, também, à ANP pela cessão dos dados do Campo Escola de Namorado.

\section{Referências bibliográficas}

ABRAHAMSEN, P., HAUGE, R. HEGGLAND, K., MOSTAD, P. Estimation of gross rock volume of filled geological structures with uncertainty measures. SPE Reservoir Eval. \& Eng. v. 3 , n. 4, p.304-309, 2000.

AI, C. A semiparametric maximum likelihood estimator. Econometrica, v. 65, n. 4, p. 933-963, 1997.

ANDREWS, D.W.K. Asymptotic normality of series estimators for nonparametric and semiparametric regression models. Econometrica, v. 59, p. 307-45, 1991.

APPLEBAUM, D. Levy Processes and Stochastic Calculus, Cambridge University Press, 2004. 408p.

BÁRDOSSY, G., FODOR, J. Traditional and new ways to handle uncertainty in geology. Natural Resources Research, v.10, n. 3, p. 179-187, 2001.

BARNDORFF-NIELSEN, O. E., MIKOSCH, T., S. RESNICK (Eds.). Levy processes: theory and applications. Birkhauser, 2001. 415p.

BHATTACHARYYA, G. K., JOHNSON, R. Statistical concepts and methods. John Willey \& Sons, 1977. 639p.

CHIERICI, G. L. Principles of petroleum reservoir engineering. Berlin: Springer-Verlag, 1994.

DEUTSCH, C. V. Geostatistical reservoir modeling. Oxford University Press, 2002.

FOWLER, M. L., YOUNG, M. A., MADDEN, M. P., COLE, E. L. The role of reservoir characterization in the reservoir management process (as reflected in the Department of Reservoir Management Demonstration Program). In: SCHATZINGER, R., JORDAN, J. (Ed.). Reservoir characterization recent advances. AAPG Memoir 71, p. 3-18, 1999.
GUARDADO, L. R., GAMBOA, L. A. P., LUCCHESI, C. F. Petroleum geology of the Campos Basin, Brazil, a model for a producing Atlantic type basin. American Association of Petroleum Geologists Memoir, 48, p. 3-79, 1990.

HÄRDLE, W, MÜLLER, M., SPERLICH, S., WERWATZ, A. Nonparametric and semiparametric models. Springer-Verlag. 2004, 300p

HEARST, J. R., NELSON P. H. Welllogging for physical properties. New York: McGraw-Hill, 1985. 492p.

HOOG, R. V., CRAIG, A. T. Introduction to mathematical statistics. Fifth edition. New Jersey: Prentice Hall, 1995. 563p.

LAKE, L. W., CARROLL, Jr., H. B. Preface. Reservoir characterization. Orlando, Florida: Academic Press, 1986. 659p.

LAW, A. M., W. D. KELTON. Simulation Modeling and Analysis. New York: McGraw-Hill Book Company, 1982. 800p.

LERCHE, I. Geological risk and uncertainty in oil exploration: uncertainty, risk and strategy. Academic Press, Inc, 1997. 658p.

LÉVY, PAUL. Calcul des probabilités. Paris: Gauthier-Villers, 1925. 352p.

MANN, C. J.Uncertainty in geology. In: DAVIS, J. C., HERZFELD, U. C. (Ed.). Computers in geology - 25 years of progress. Oxford: Oxford Univ. Press, 1993. p. 241-254.

SALOMÃO, M. C., GRELL, A. P. Uncertainty in production profiles on the basis of geostatistic characterization and flow simulation. SPE Latin American and Caribbean Petroleum Engineering Conference. Buenos Aires, Argentina, 25-28 March. SPE 69477, 2001.

SCHLUMBERGER. Log interpretation: principles/applications. Houston TX: Educational Services, 1987.

Artigo recebido em 07/11/2005 e aprovado em 10/05/2006.

\section{REM - Revista Escola de Minas www.rem.com.br}

